\title{
The distributed practice effect and list length in paired-associate learning'
}

\author{
DAVID C. RIMM, Department of Psychology, Arizona State \\ University, Tempe, Arizona 85281
}

The hypothesis that distributed practice would facilitate paired associate learning for short lists but not for long lists was investigated. Ss were run on lists containing 3, 6, or 9 pairs, with intertrial intervals of 0,4 , or 10 sec. The results did not confirm the hypothesis. The question of why Ss did not appear to benefit from the rehearsal possible over longer intertrial intervals was raised. A tentative hypothesis, related to trial to trial variation in order of pairs was offered as a possible answer.

Nodine \& Nodine (1966), Nodine, Nodine, \& Thomas (1967) and Rimm \& Biggs (1967) have shown that in paired-associate learning, increasing the interpair interval facilitates learning. These authors have presented evidence supporting the view that rehearsal may mediate such facilitation. When Ss were required to engage in digit naming or backward counting (Peterson \& Peterson, 1959) over the interpair intervals ranging from 2 to $5 \mathrm{sec}$ performance was significantly inferior to performance when the interpair interval was unfilled. It seemed reasonable to assume that $S$ rehears during this unfilled interval and that this accounts for the superior performance.

On the other hand, it seems to be rather well established that increasing intertrial rather than interpair interval results in very little improvement in paired associate learning (Underwood \& Ekstrand, 1967). One possible explanation for the failure to find facilitation under distributed practice lies in the relatively long lists (e.g., six to eight pairs per list) typically employed. The amount of information contained in lists of such length might exceed S's immediate memory span, or for some other reason rehearsal for long lists might be cumbersome, resulting in S's not even attempting to rehearse. If long lists do discourage rehearsal one might expect to obtain a negative relationship between length of list and facilitation in paired-associate learning through distributed practice.

In order to investigate the relationship between the effect of distribution of practice and list length, Ss were required to learn lists containing 3,6, or 9 pairs. For each list length, intertrial interval was either 0,4 , or $10 \mathrm{sec}$. The hypothesis under investigation was that distributed practice ( 4 and $10 \mathrm{sec}$ intervals) would result in the most facilitation when the list was three pairs in length and least when the list was nine pairs in length.

\section{METHOD}

The Ss were 90 students, both male and female, enrolled in the introductory psychology course at Arizona State University; participation fulfilled a course requirement. The stimulus items were presented via an Airequipt 420 slide projector. Stimuli were CVC trigrams each having an association value of $53 \%$ (Glaze, 1928).

The Ss were required to learn which of three response alternatives (saying aloud "one," "two," or "three") was associated with each nonsense syllable. The same three response alternatives were employed for each list length so as to eliminate possible effects resulting from differences in response availability as well as differences in the probability of guessing correctly. One group of $30 \mathrm{Ss}$ was required to learn three pairs, a second group, six pairs, and a third group of $30 \mathrm{Ss}$ was required to learn a list containing nine pairs, all to a criterion of two consecutive errorless trials. At the beginning of each trial a stimulus item was projected on the wall in front of S. Immediately following S's response S was told which response had been correct, and the next stimulus was presented. This procedure was continued until $S$ had completed the list. Order of syllables was varied randomly from trial to trial. Ten Ss in each list length condition were run under massed practice, with a $0 \mathrm{sec}$ delay between successive trials. This meant that immediately following E's correction of S's response to the last item of a given trial, the first stimulus of the next trial was presented. Ten Ss in each list length condition were run under disturbed practice with a 4-sec delay between the correction of the last response on a given trial and presentation of the first stimulus of the succeeding trial. Finally, $10 \mathrm{Ss}$ in each list length condition were run under distributed practice with a 10 -sec delay between successive trials.

\section{RESULTS AND DISCUSSION}

For lists of three pairs, mean trials to criterion for the intertrial intervals of 0,4 , and $10 \mathrm{sec}$ were respectively $1.2,1.7$, and 3.8 . Comparable mean trials to criterion for lists containing six pairs were 14.7, 13.7, and 14.0, and for nine pair lists, 14.0,21.0, and 17.7. A two way analysis of variance revealed a highly significant list length effect $[F(2,81)=64.12, p<.001]$. However, neither the intertrial interval effect $[F(2,81)=1.37, p>.10]$ nor the list length by intertrial interval interaction $[F(4,81)=1.70, p>.10]$ approached statistical significance.

For three pair lists, mean errors to criterion for the intervals of 0,4 , and 10 sec were respectively $1.4,2.2$, and 4.6. Mean errors to criterion for six pair lists were $37.3,35.8$, and 34.0 , and for the nine pair lists, 48.7, 68.7, and 58.3. The analysis of variance again revealed a highly significant effect of list length $[F(2,81)=95.5$, $p<.001]$ but again, neither the effect of varying intertrial interval $[F(2,81)=1.32, \quad p>.10]$ nor the interaction effect $[F(4,81)=1.47, p>.10]$ approached significance.

Inspection of the data suggests the possibility that a real intertrial interval effect may in fact have been in operation for three-pair lists, but that the means under this condition (both trials and errors) were so small that whatever differences obtained between them could not be expected to contribute appreciably to the overall interaction variance. Therefore one-way analyses of variance were performed on scores based upon three pair lists only. For trials to criterion, the effect of interpair interval was found to be significant $[F(2,27)=4.52, p<.05]$. For errors to criterion, the effect was also found to be significant $[F(2,27)=5.25, p<.05]$. The post hoc nature of these particular analyses weakens somewhat any interpretation of results. Nevertheless, it should be noted that for three pair lists, increasing the interpair interval appears to have inhibited, rather than facilitated paired associate learning.

These results may be taken as offering additional support for the view (Underwood \& Ekstrand, 1967) that distributed practice has little or no facilitative effect on paired associate learning. This appears to be the case for short lists (three pairs in length) as well as relatively long lists (nine pairs in length).

Studies using paired associates in which interpair rather than intertrial interval was manipulated (e.g., Nodine, Nodine, \& Thomas, 1967; Rimm \& Biggs, 1967) have found noticeable "distributed practice" facilitation with rest intervals on the order of only $5 \mathrm{sec}$. If the rehearsal interpretation given to these findings is correct, then it is somewhat puzzling that Ss in the present experiment who were run on the shorter lists (especially three pairs) were unable to benefit at all from rehearsal over intertrial intervals of as great as 10 sec.

In general it would appear that failure to obtain distributed practice facilitation in paired associate learning is not simply an artifact of long lists typically employed. If Ss are inclined to rehearse over intertrial intervals, one possible factor contributing to their failure to benefit from such rehearsal might be the variations in pair order from trial to trial. Thus it is possible that Ss tend to rehearse ordered sequences of pairs, rather than pairs in isolation. Rehearsal of a given sequence of stimulus response pairs, followed by the presentation of a different sequence of the same stimulus response pairs on the next trial might result in confusion which would tend to negate whatever benefit might have accrued from such rehearsal. 
REFERI: VCES

GLAZE, J. A. The association of valuc of nonsense syllables. Journal of Genetic Psychology, 1928, 35, 255-269.

NODINE, C. F., \& NODINE. B. F. Stimulıs intervals, stimulus durations and difficulty level in paired-assiciates lcurnung. Journal of Experimental Psychology, 1966, 72, 156-158.

NODINE, C. F.. NODINE. B. F., \& THOMAS, R. C. Temporal variables in paired-assuciates learning: The roles of repetition and number tracking during stimulus intervals. Journal of Experimental Psychology. 1967, 73. 439-445.
PITI RSON I R. \& PETIRSON. M. J. Short term retemtion of ialivelual

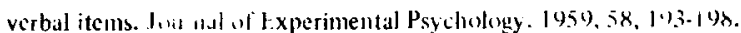

RINIM. D. C.. \& BIGGS, B. Acquisition of paired associates as a function of interpair interval. Psychonomic Science. 1967, 9, 611.612.

UNDERWOOD. B. J. \& EKSTRAND, B. R. Effect of distributed practice on. paired-associate learning. Journal of Experimental Psychology, 1967, 73 (Monographs Supplement. Whole No. 634).

$$
\text { NOTE }
$$

1. The author gratefully acknowledges assistance given him by Miss Stephanie Cranmer in the running of Ss. 